\title{
Preconditioning but not postconditioning treatment with resveratrol substantially ameliorates post-resuscitation myocardial dysfunction through the PI3K/Akt signaling pathway
}

\author{
HAIHONG ZHANG, QINQIN WU, ZHI WAN, YU CAO and ZHI ZENG \\ Emergency Department, West China Hospital, Sichuan University, Chengdu, Sichuan 610041, P.R. China
}

Received November 26, 2018; Accepted April 24, 2019

DOI: $10.3892 / \mathrm{mmr} .2019 .10318$

\begin{abstract}
Post-resuscitation myocardial dysfunction (PRMD) is a severe complication that arises in patients after cardiac arrest (CA). However, there are no safe or effective treatment strategies that are currently available to treat these patients. In the present study, it was investigated whether resveratrol administration could inhibit myocardial nitrative stress to alleviate PRMD. CA was induced in Sprague-Dawley rats by trans-oesophageal alternating electrical stimulation, followed by cardiopulmonary resuscitation. Rats were then randomly divided into a preconditioning or a postconditioning group. Left ventricular function $\left(+\mathrm{dP}^{\mathrm{d}} \mathrm{dt}_{\max }\right.$ and $\left.-\mathrm{dP}^{\mathrm{d}} / \mathrm{dt}_{\min }\right)$ was recorded for $4 \mathrm{~h}$ after the return of spontaneous circulation (ROSC), after which the animals were euthanized. Myocardial nitrative stress was analysed using enzyme-linked immunosorbent assay, western blotting and immunohistochemistry. Wortmannin (a PI3K inhibitor) was used to investigate the involvement of the PI3k/Akt signalling pathway in the cardio-protective activity of resveratrol. After ROSC, resveratrol improved PRMD compared to the vehicle control; however, resveratrol administration significantly improved PRMD in the preconditioning group compared to the postconditioning group. Likewise, resveratrol preconditioning significantly decreased the expression of iNOS and nitrotyrosine in rat hearts but did not significantly ameliorate myocardial nitrative stress. Wortmannin partially inhibited the protective effect of resveratrol preconditioning and resulted in the deterioration of cardiac function and increase in iNOS and nitrotyrosine levels. Resveratrol preconditioning could alleviate PRMD by inhibiting myocardial nitrative stress. The PI3K/Akt signalling pathway may be partially involved in the process.
\end{abstract}

Correspondence to: Dr Zhi Zeng, Emergency Department, West China Hospital, Sichuan University, 37 Guo Xue Street, Chengdu, Sichuan 610041, P.R. China

E-mail: zengzhi@cd120.com

Key words: cardiac arrest, cardiopulmonary resuscitation, resveratrol, nitrative stress

\section{Introduction}

Cardiac arrest (CA) is a major cause of mortality worldwide. There are approximately 544,000 cases of CA each year in China (1). Although the preliminary rate of return of spontaneous circulation (ROSC) is $40-50 \%$, the majority of CA survivors succumb to this event before discharge, resulting in less than $10 \%$ in-hospital survival rate (2). High in-hospital mortality in the early stages after ROSC is most often associated with multisystem organ failure due to myocardial dysfunction $(3,4)$. Although a great amount of effort has been made to improve PRMD, no treatment, including drugs that have demonstrated translational efficacy in animal models, have exhibited clinical efficacy in humans to date.

The pathogenesis of PRMD is multifactorial and complex. To date, the mechanism underlying its pathogenesis remains to be deciphered. In rodent CA models, after ROSC, there were significant reductions in the activity of mitochondrial complex I, a marked production of ROS, mitochondrial protein oxidation, and tyrosine nitrosylation (5). Several studies have suggested that nitric oxide (NO)-derived reactive nitrogen species (RNS) contributes to pathologic tissue injury by nitrative protein modification (nitrative stress). This may serve as a possible target for therapeutic intervention $(6,7)$. $\mathrm{NO}$ is produced from the conversion of L-arginine by nitric oxide synthase (NOS). Previous studies have demonstrated that a high pathological concentration of NO, produced by inducible NOS (iNOS), results in nitrative stress and tissue injury (8). $\mathrm{NO}$ is not toxic and does not cause significant tissue injury even at high concentrations; however, $\mathrm{NO}$ and superoxide can combine in a diffusion-limited reaction to generate peroxynitrite $\left(\mathrm{ONOO}^{-}\right)$, resulting in nitrative molecules that are toxic (9). Studies have demonstrated that $\mathrm{ONOO}^{-}$plays a critical role in myocardial ischemia/reperfusion injury (10). Hence, drugs that suppress $\mathrm{ONOO}^{-}$formation or antagonize its activity may protect the heart from reperfusion injury (11). However, it is not clear whether nitrative stress is involved in PRMD or whether inhibitors of nitrative stress could alleviate PRMD.

Resveratrol is a natural polyphenol phytoalexin that is present in numerous plant species and is thought to be responsible for the 'French paradox'. Several studies have demonstrated that resveratrol has beneficial effects in several cardiovascular diseases. These benefits may be due to its 
versatile biological effects such as attenuation of oxidative and nitrative stress, which are involved in the pathophysiologic mechanisms of PRMD $(12,13)$. In a rat model of local myocardial ischaemia-reperfusion, resveratrol preconditioning inhibited excessive NO expression that resulted after reperfusion (14). However, to date, there are limited studies regarding the use of resveratrol in post-resuscitation models.

Preconditioning and postconditioning with resveratrol have revealed beneficial effects in some local ischemia studies $(15,16)$. Resveratrol had a dose-dependent effect in the previous studies, where low doses $(5-20 \mu \mathrm{M})$ protected cells while higher doses $(10-40 \mathrm{mM})$ induced apoptosis $(17,18)$. However, these results have not been ascertained in CA models, where the optimal dose is unknown.

A rat $\mathrm{CA}$ model was used to determine whether resveratrol could attenuate PRMD via its inhibitory effect on myocardial nitrative stress.

\section{Materials and methods}

Ethical statement. The present study was performed in strict accordance to the recommendations by the Guide for the Care and Use of Laboratory Animals, National Institutes of Health. The Institutional Animal Care and Use Committee of West China Hospital (Sichuan University; approval no 2016045A) approved the study protocol. All surgeries were performed under sodium pentobarbital anaesthesia and every effort was made to minimize suffering.

Animals and preparatory procedures. Specific pathogen-free Sprague-Dawley (SD) male rats 12 weeks of age and weighing $350-400 \mathrm{~g}$ were used for the study. The animals were fasted on the night before the procedure but were allowed free access to water. The animals were prepared as previously described $(19,20)$. Briefly, rats were anaesthetized with intra-peritoneal injections of $45 \mathrm{mg} / \mathrm{kg} 1.5 \%$ pentobarbital, placed in a supine position and immobilized. They were then intubated with a standard endotracheal tube and anaesthesia was maintained with $1.5 \%$ pentobarbital at $15 \mathrm{mg} / \mathrm{kg} / \mathrm{h}$ intraperitoneally. The rats were mechanically ventilated using a volume-controlled ventilator (HX-100E; Chengdu TME Technology \& Market Co., Ltd.) with a tidal volume of $6 \mathrm{ml} / \mathrm{kg}$ and a fraction of inspired oxygen (FiO2) of 1.0 (21). Electrocardiograms (ECGs) were monitored with the aid of subcutaneous needles throughout the procedure. A $24 \mathrm{G}$ catheter was introduced into the right femoral artery to measure arterial blood pressure, while drug infusion was performed through a $20 \mathrm{G}$ catheter inserted into the right femoral vein. A saline-filled PE-50 catheter (BD Biosciences) was advanced from the right carotid artery into the left ventricle cavity for measurement of left intraventricular pressure. The ECG, arterial blood pressure and left intraventricular pressure were monitored continuously via a 4-channel physiological recorder (BL-420F Data Acquisition \& Analysis System; Chengdu TME Technology Co., Ltd.).

Experimental protocol and grouping. A pacing electrode (model 3830; Medtronic, Inc.) with two 1-mm ring electrodes and an inter-electrode distance of $5 \mathrm{~mm}$ was inserted orally into the oesophagus to a depth of $4 \mathrm{~cm}$. After a $30-\mathrm{min}$ stabilization period, CA was induced via an oesophageal electrode connected to a cardiac electrophysiological stimulation apparatus (BL-420F Data Acquisition \& Analysis System). The current had an amplitude of $6 \mathrm{~mA}$, with a frequency of $50 \mathrm{~Hz}$, and a wave width of $10 \mathrm{msec}$ (19).

CA was defined as an ECG having waveforms of ventricular fibrillation, pulseless electricity activity, or asystole with a rapid decline in systolic pressure to below $25 \mathrm{mmHg}$. Artificial ventilation was paused while inducing CA. Cardiopulmonary resuscitation was initiated after $5 \mathrm{~min}$ of CA. A mechanical chest compressor (College of Electrical Engineering, Sichuan University) with artificial ventilation of 80 breaths $/ \mathrm{min}$, tidal volume of $6 \mathrm{ml} / \mathrm{kg}$, and $\mathrm{FiO}_{2}$ of 1.00 was used to deliver 200 chest compressions/min. Epinephrine was administered $(20 \mu \mathrm{g} / \mathrm{kg})$ immediately and one dose every $5 \mathrm{~min}$ after the compressions. After the first $5 \mathrm{~min}$, a $2 \mathrm{~J}$ electrical shock was delivered if ventricular fibrillation was present. Subsequently, an electrical shock was delivered every $1 \mathrm{~min}$ if necessary, however, the number of shocks were no more than 3 times in the whole experiment. ROSC was characterized by spontaneous cardiac rhythms with a mean arterial pressure (MAP) $\geq 60 \mathrm{mmHg}$ and persisting for more than $10 \mathrm{~min}$. Rats were included for further study if ROSC persisted for at least $30 \mathrm{~min}$. Left ventricular function $\left(+\mathrm{dP} / \mathrm{dt}_{\max }\right.$ and $\left.-\mathrm{dP} / \mathrm{dt}_{\min }\right)$ was recorded at $0.5,1,2,3$ and $4 \mathrm{~h}$ after ROSC, after which the animals were euthanized and their hearts were rapidly removed for analyses. Post-resuscitation care was similar for all rats in the different groups. Sham control animals were similarly treated except they received ventilation without CA induction (Fig. 1A and B). In the experiment, all rats were sacrificed by cervical dislocation under anesthetic conditions maintained by $1.5 \%$ pentobarbital at $15 \mathrm{mg} / \mathrm{kg} / \mathrm{h}$ intraperitoneally. All experiments were performed at room temperature.

Animals which met the inclusion criteria as stated before were randomly assigned to one of two groups: A preconditioning or postconditioning group, based on different intervention times. Prior to administration, resveratrol (Sigma Aldrich; Merck KGaA) was dissolved in dimethyl sulfoxide (DMSO) and then in sterile water containing $0.01 \%$ DMSO. Resveratrol or vehicle was administered by bolus injection $15 \mathrm{~min}$ before CA to the preconditioning group and $1 \mathrm{~min}$ before CPR to the postconditioning group. Each group was then subdivided into four groups (6 rats/group) and administered different doses of resveratrol: i) Vehicle (DMSO); ii) $2.3 \mathrm{mg} / \mathrm{kg}$ resveratrol; iii) $0.23 \mathrm{mg} / \mathrm{kg}$ resveratrol, and iv) $0.023 \mathrm{mg} / \mathrm{kg}$ resveratrol (Fig. 1C). Resveratrol doses that were administered were based on data from several studies on the aqueous solubility of resveratrol $(12,22)$. The 6 rats in the sham group underwent surgical procedures without induced cardiac arrest and showed no differences between thepreconditioning and postconditioning groups.

Based on the preliminary results, the resveratrol group that exhibited the greatest significant myocardial protective effect was selected and it was investigated whether wortmannin (Sigma Aldrich; Merck KGaA) inhibited its protective effect. This part of the study comprised of four groups (6 rats/group): i) Sham; ii) vehicle; iii) resveratrol; iv) resveratrol $+15 \mu \mathrm{g} / \mathrm{kg}$ wortmannin (Rev+Wom). Resveratrol and wortmannin were administered simultaneously to the 4-h group. 

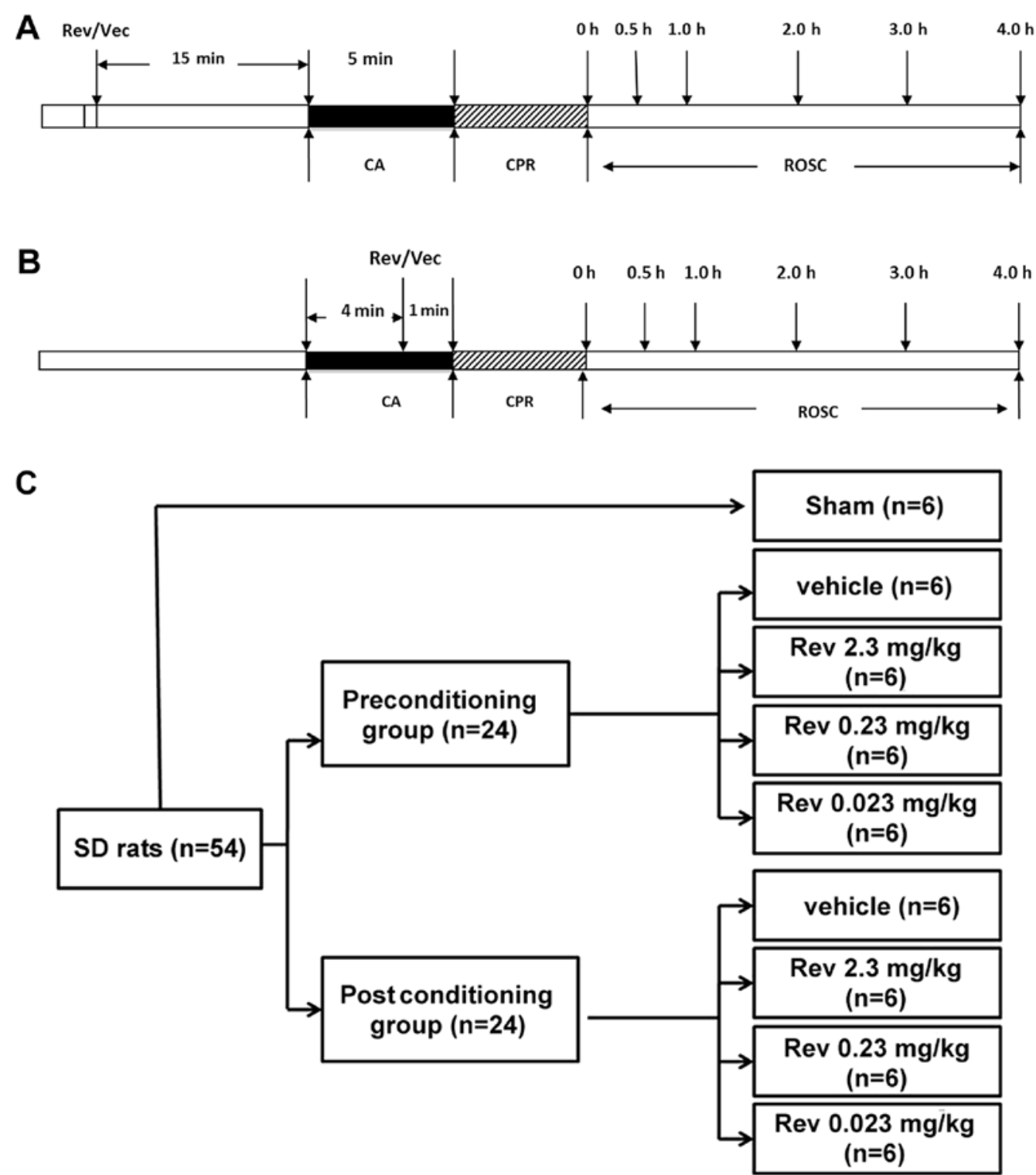

Figure 1. Experimental protocol. SD rats were anesthetized and stabilized for $30 \mathrm{~min}$ before intervention. Rats were monitored for $4 \mathrm{~h}$ after ROSC. (A) Preconditioning group. Resveratrol or vehicle was administered 15 min prior to CA. CA was maintained for 5 min, after which CPR was initiated. (B) Post-conditioning group. Resveratrol or vehicle was administered 1 min before CPR during CA. (C) Schematic for study grouping. Rev, resveratrol; Vec, vehicle; CA, cardiac arrest; CPR, cardiopulmonary resuscitation; ROSC, return of spontaneous circulation.

Histological examination of heart sections. Left ventricular tissues were fixed in $4 \%$ buffered formalin for $20 \mathrm{~min}$ at room temperature. After embedding in paraffin, the tissues were sliced into $4-\mu \mathrm{m}$ sections and stained for nitrotyrosine by the following protocol. The sections were deparaffinized and hydrated using decreasing gradients of ethanol followed by antigen retrieval. The sections were then incubated in $0.3 \% \mathrm{H}_{2} \mathrm{O}_{2}$ in phosphate-buffered saline (PBS) to block endogenous peroxidase activity. The sections were then incubated with anti-nitrotyrosine $(1: 1,000 ; 189542-50$; Cayman Chemical) antibody at $4{ }^{\circ} \mathrm{C}$ overnight in a moist chamber. Goat anti-mouse Biotinylated secondary antibodies (1:1,000; 10006617; Cayman Chemical) were then added to the tissues according to the manufacturer's instructions and then stained with DAPI (Sigma Aldrich; Merck KGaA) at room temperature for 3-5 min. Subsequently, the sections were dehydrated in ethanol, cleared in xylene and mounted for viewing. Stained sections were visualized and images were acquired using an IX-71 fluorescent microscope (Olympus Corporation). Histological evaluation was performed in a blinded manner.

Expression levels of iNOS, Akt, and phosphorylated-Akt $(p-A k t)$. Left ventricle tissue samples were lysed using radioimmunoprecipitation assay buffer (Beyotime Institute of Biotechnology) containing a Protease Inhibitor Cocktail (Promega Corporation) for $30 \mathrm{~min}$ on ice, and after sonication were centrifuged for $10 \mathrm{~min}$ at $4^{\circ} \mathrm{C} 12,000 \mathrm{x} \mathrm{g}$. Equal amounts of protein (30 $\mu \mathrm{g}$ protein/lane) were separated by electrophoresis on a $10 \%$ sodium dodecyl sulphate polyacrylamide gel (SDS-PAGE) and transferred onto polyvinylidene difluoride membranes (EMD Millipore). After blocking with 5\% skim milk in Tris-buffered saline at room temperature $\left(18-28^{\circ} \mathrm{C}\right)$ for $1 \mathrm{~h}$, the membranes were incubated with antibodies $(1: 1,000)$ against iNOS (ab49999; Abcam), Akt 

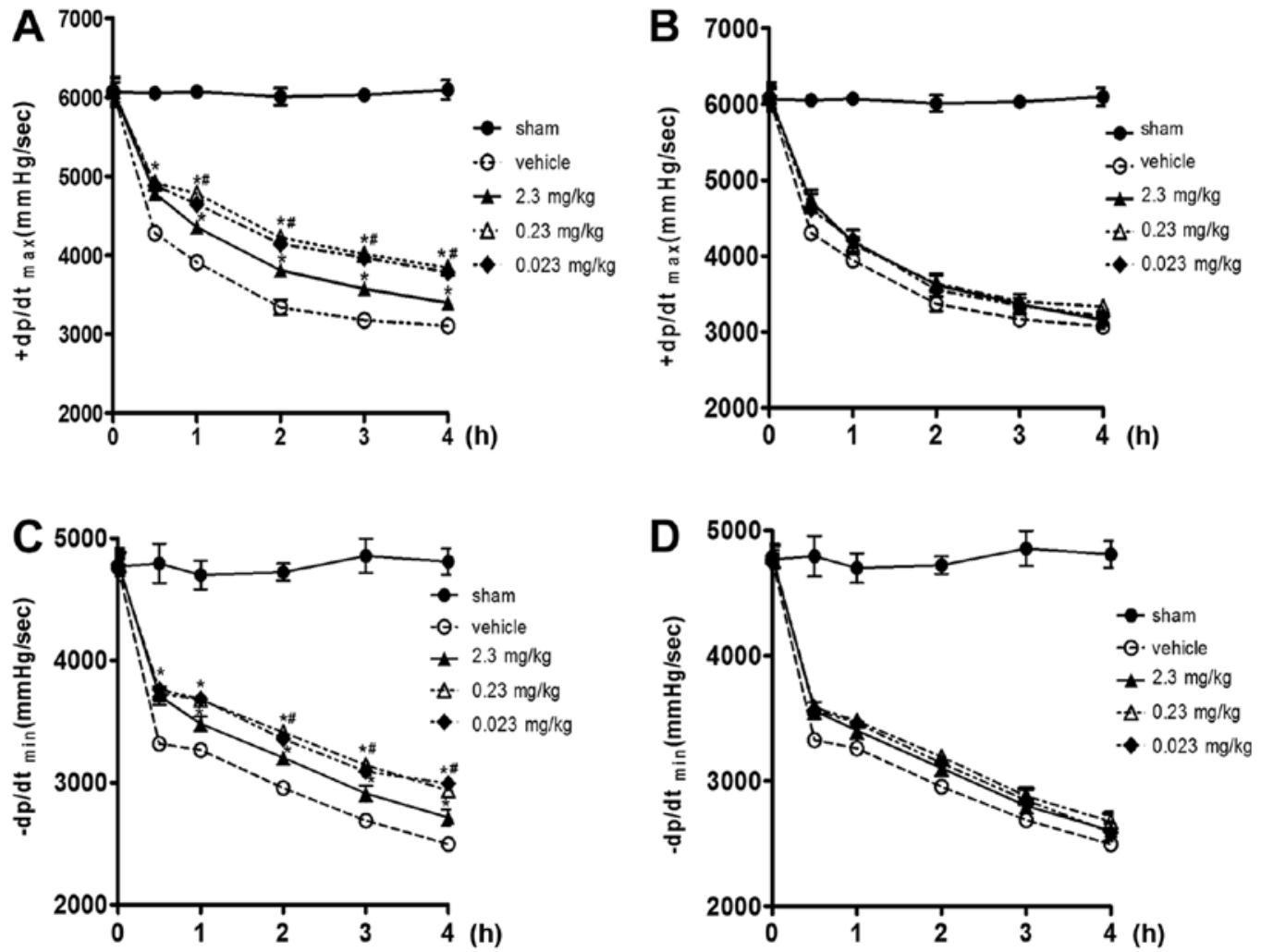

Figure 2. Left ventricular function variables for the experimental groups. (A and C) Preconditioning group. (B and D) Postconditioning group.

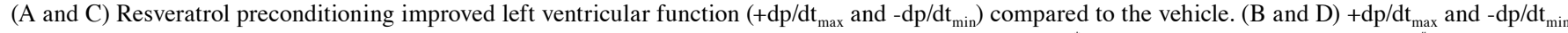
in the resveratrol postconditioning group were not significantly different compared to the vehicle group ( $\mathrm{P}<0.05$ vs. the vehicle group and ${ }^{*} \mathrm{P}<0.05$ vs. the $2.3 \mathrm{mg} / \mathrm{kg}$ group).

(9272), or p-Akt (4051; both Cell Signaling Technology, Inc.) at $4^{\circ} \mathrm{C}$ overnight. The membranes were then washed with phosphate-buffered saline with Tween-20 and incubated with horseradish peroxidase-conjugated $\operatorname{IgG}$ antibody $(1: 1,000$; 7076; Cell Signaling Technology, Inc.) at $37^{\circ} \mathrm{C}$ for $1 \mathrm{~h}$. Blots were developed using an enhanced chemiluminescence detection kit (Pierce Biotechnology; Thermo Fisher Scientific, Inc.). The proteins were visualized using ChemiDoc XRS and band densities were analysed using Quantity One software 4.6 (both from Bio-Rad Laboratories, Inc.).

Quantitation of myocardial nitrotyrosine levels by enzyme-linked immunosorbent assay (ELISA). Cardiac nitrotyrosine, a determinant for in vivo RNS formation, was measured using ELISA as previously reported (18). In brief, nitrotyrosine tissue levels were measured using a Nitrotyrosine ELISA kit (Chemiluminescence Detection kit; EMD Millipore) in accordance with the manufacturer's instructions and expressed as $\mathrm{nmol} / \mathrm{g}$ protein.

Immunofluorescence measurements. Paraformaldehyde-fixed myocardial tissues were cut into semi-thin sections, of 4-5 $\mu \mathrm{m}$ thickness, and stained with a primary antibody $(1: 1,000)$ against nitrotyrosine (189542; Cayman Chemical) at room temperature for $1 \mathrm{~h}$ in a wet box and then at $4^{\circ} \mathrm{C}$ overnight, followed by incubation with the goat anti-mouse secondary antibody (1:1,000; ZK-9600; OriGene Technologies, Inc.) at $37^{\circ} \mathrm{C}$ for $30 \mathrm{~min}$ and then counterstained with DAPI for 10 min at $37^{\circ} \mathrm{C}$ (Sigma Aldrich; Merck KGaA).
Statistical analysis. All data were expressed as the mean \pm standard deviation. Statistical significance was determined using one-way analysis of variance (ANOVA) followed by the Student-Newman-Keuls' method using SPSS 19.0 (IBM Corp.) and GraphPad Prism 5.0 software (GraphPad Software, Inc.). $\mathrm{P}<0.05$ was considered statistically significant (two-tailed).

\section{Results}

Animals. A total of 54 rats were used in this study. All rats attained ROSC and were sustained for over $30 \mathrm{~min}$.

Left ventricular function. The left ventricular variables $\left(+\mathrm{dp} / \mathrm{dt}_{\max }\right.$ and $\left.-\mathrm{dp} / \mathrm{dt}_{\min }\right)$ for all post-resuscitation animals were reduced after ROSC until the end of the procedure. From 0.5 to $4 \mathrm{~h}$ after ROSC, resveratrol preconditioning improved left ventricular function significantly compared to vehicle $(\mathrm{P}<0.05)$. The effect of resveratrol on left ventricular function was significant at doses between 0.23 and $0.023 \mathrm{mg} / \mathrm{kg}$, but not at $2.3 \mathrm{mg} / \mathrm{kg}(\mathrm{P}<0.05 ;$ Fig. $2 \mathrm{~A}$ and $\mathrm{C})$. During the whole procedure, resveratrol improved $+\mathrm{dp} / \mathrm{dt}_{\max }$ and $-\mathrm{dp} / \mathrm{dt}_{\min }$ in the postconditioning group compared to the vehicle, however, the observed differences were not significant $(\mathrm{P}>0.05$; Fig. 2B and D).

iNOS expression in heart tissue. Then, $4 \mathrm{~h}$ after ROSC, iNOS expression in the vehicle groups was significantly increased compared to the sham groups $(\mathrm{P}<0.05)$. However, the iNOS levels in the preconditioning groups were significantly lower 

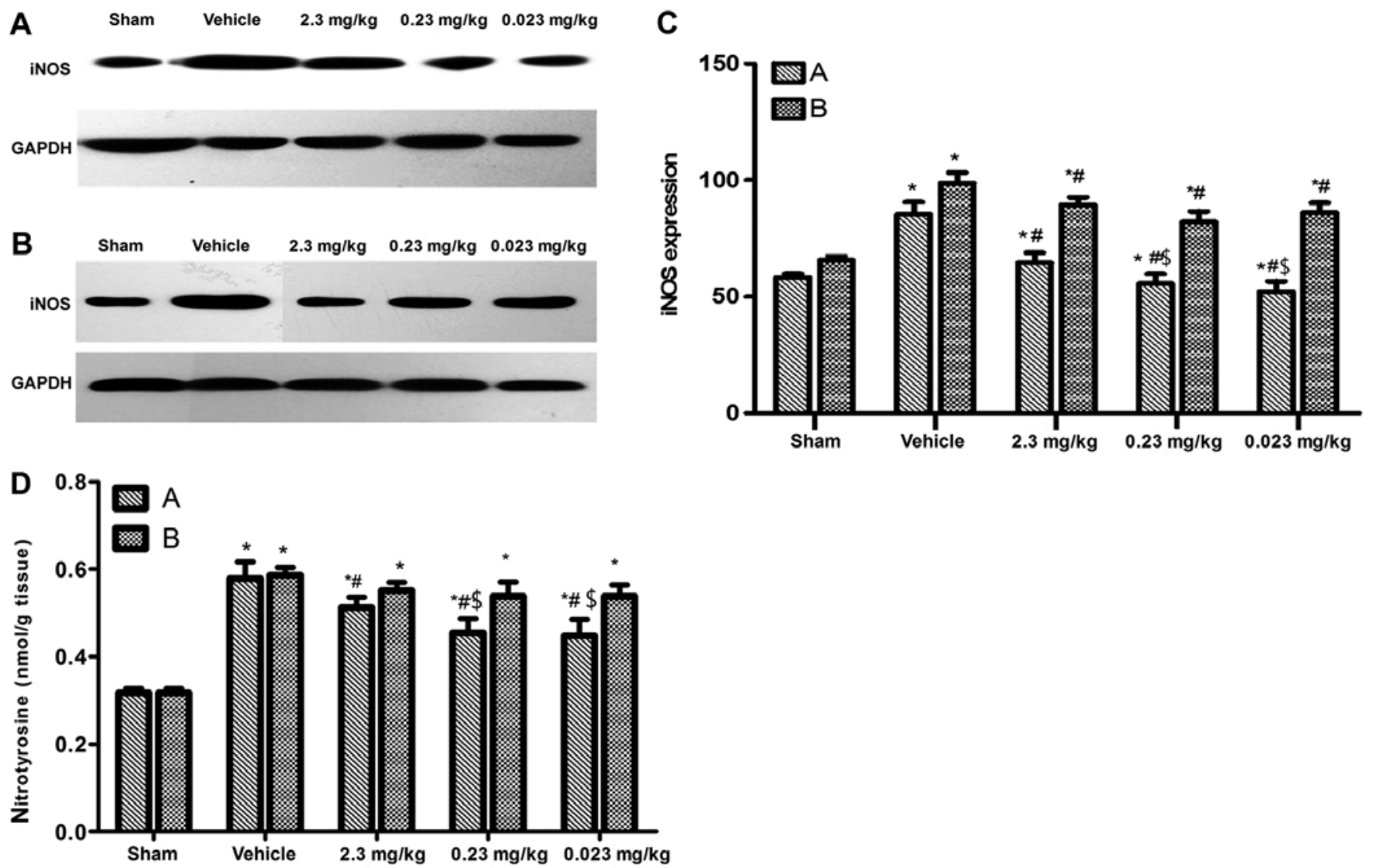

Figure 3. iNOS and nitrotyrosine expression in heart tissues. Data are presented as the mean \pm standard deviation. (A) Resveratrol preconditioning. (B) Resveratrol postconditioning. A: Representative blots of iNOS in heart tissues from rats administered different doses of resveratrol in preconditioning groups. B: Representative blots of iNOS in heart tissues from rats administered different doses of resveratrol in the postconditioning groups. (C) Statistical data obtained from quantitative densitometric analysis of iNOS blots. Sham group, $\mathrm{n}=3$; other groups, $\mathrm{n}=6$. ${ }^{*} \mathrm{P}<0.05$ vs. sham, ${ }^{\prime \prime} \mathrm{P}<0.05$ vs. vehicle, ${ }^{\text {S }} \mathrm{P}<0.05$ vs the $2.3 \mathrm{mg} / \mathrm{kg}$ group. (D) Statistical data obtained for quantitative RLU of nitrotyrosine levels obtained by ELISA. "P<0.05 vs. the sham group, ${ }^{*} \mathrm{P}<0.05$ vs. the vehicle group and ${ }^{\mathrm{S}} \mathrm{P}<0.05$ vs. the $2.3 \mathrm{mg} / \mathrm{kg}$ group. RLU, relative light units; iNOS, inducible NOS.

compared to the vehicle group $(\mathrm{P}<0.05)$, while the 0.23 and $0.023 \mathrm{mg} / \mathrm{kg}$ administered groups were not significantly different compared to the sham group $(\mathrm{P}>0.05)$. The iNOS levels in the postconditioning groups were lower compared to the vehicle group $(\mathrm{P}<0.05)$, but were higher compared to the sham group $(\mathrm{P}<0.05$; Fig. 3A-C).

Nitrotyrosine expression in heart tissue. Four hours after ROSC, the expression of nitrotyrosine in the vehicle group was significantly higher compared to the sham group $(\mathrm{P}<0.05)$. Nitrotyrosine levels in the preconditioning groups were significantly lower compared to the vehicle group $(\mathrm{P}<0.05)$, especially in the 0.23 and $0.023 \mathrm{mg} / \mathrm{kg}$ administered groups. However, resveratrol postconditioning did not significantly decrease the nitrotyrosine expression levels compared to the vehicle ( $\mathrm{P}>0.05$; Figs. 3D and 4).

Role of the PI3K/Akt signalling pathway in the myocardial protective effect of resveratrol; wortmannin prevents the protective effects of resveratrol in PRMD. From $0.5 \mathrm{~h}$ after ROSC until the end of the procedure, the left ventricular function variables $\left(+\mathrm{dp} / \mathrm{dt}_{\max }\right.$ and $\left.-\mathrm{dp} / \mathrm{dt}_{\min }\right)$ in the resveratrol $(0.23 \mathrm{mg} / \mathrm{kg})+$ wortmannin group were lower compared to the resveratrol $(0.23 \mathrm{mg} / \mathrm{kg})$ group $(\mathrm{P}<0.05)$, but higher compared with the vehicle group $(\mathrm{P}<0.05)$. Wortmannin partially inhibited the beneficial effects of resveratrol on left ventricular function (Fig. 5).

Preliminary results revealed that resveratrol preconditioning ameliorated myocardial nitrative stress after ROSC. Administration of resveratrol+wortmannin had higher iNOS expression levels compared to resveratrol alone $(\mathrm{P}<0.05)$; however, iNOS expression levels in the resveratrol+wortmannin and resveratrol groups were lower compared to the vehicle group $(\mathrm{P}<0.05$; Figs. 6 and 7$)$. These results indicated that wortmannin partially reversed the inhibitory effect of resveratrol on nitrative stress. p-Akt levels in the resveratrol group were higher compared to the sham group and the vehicle group, while wortmannin decreased p-Akt levels. These differences were statistically significant $(\mathrm{P}<0.05)$. No significant differences in Akt levels were observed for the four groups (Fig. 6).

\section{Discussion}

In the present study, resveratrol had diverse effects on myocardial function after cardiac arrest. This effect was dose- and time-dependent. Resveratrol preconditioning improved myocardial function after $\mathrm{CA}$, but not when administered post CA. Lower doses $(0.23,0.023 \mathrm{mg} / \mathrm{kg})$ had superior efficacy compared to higher doses of resveratrol. 


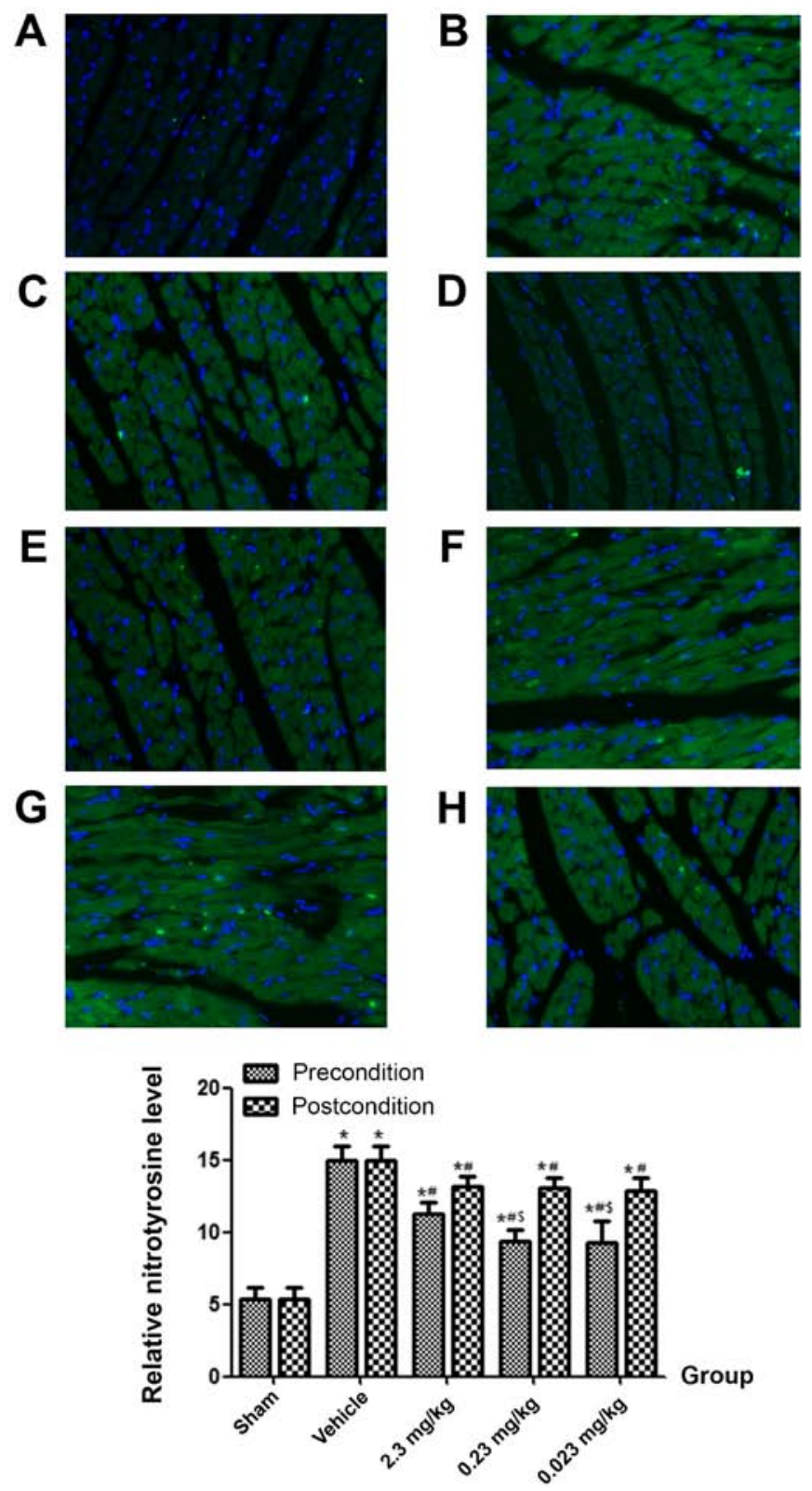

Figure 4. Representative photomicrographs of in situ detection of nitrotyrosine expression levels and DAPI staining in rat heart tissues (inverted phase contrast microscope, $\mathrm{x} 400$ ). Blue fluorescence indicates nuclei of cardiomyocytes. Green fluorescence indicates nitrotyrosine in the cytoplasm. (A) Sham; (B) vehicle; (C-E) resveratrol preconditioning group $(2.3,0.23$ and $0.023 \mathrm{mg} / \mathrm{kg}$, respectively); and (F-H) resveratrol postconditioning group (2.3, 0.23 and $0.023 \mathrm{mg} / \mathrm{kg}$, respectively). Nitrotyrosine expression increased after ROSC. Resveratrol preconditioning significantly decreased nitrotyrosine expression levels while resveratrol postconditioning had no effect. ${ }^{*} \mathrm{P}<0.05$ vs. the sham group, ${ }^{\sharp} \mathrm{P}<0.05$ vs. the vehicle group and ${ }^{\$} \mathrm{P}<0.05$ vs. the $2.3 \mathrm{mg} / \mathrm{kg}$ group. ROSC, return of spontaneous circulation.

As the results revealed, resveratrol preconditioning improved left ventricular function after CA, which was similar to certain previous studies $(16,23)$. Given the sudden onset and unpredictability of CA, the protocol in the present study appears infeasible in clinical practice, however, oral resveratrol for a short period of time maybe practicable, as a previous study reported, this however requires validation in the future (24). In the present study resveratrol, postconditioning did not significantly ameliorate myocardial dysfunction after CA which was not similar to previous research. In an in vitro ischemia-reperfusion rat model, $1 \mu \mathrm{M}$ or $10 \mu \mathrm{M}$ resveratrol administered during the reoxygenation period after recovery from ischemia improved left ventricular contractile function and attenuated myocardial injury (16). In another local myocardial ischemia-reperfusion rat study, $10 \mu \mathrm{M}$ resveratrol administered during the ischemic period and before perfusion improved myocardial infarct size and mitochondrial injury (15). The present study did not demonstrate a significant postconditioning effect of resveratrol after $\mathrm{CA}$, which may be due to the following reasons. First, the effect of resveratrol on the induction of mRNA transcript levels may take a longer time resulting in delayed protein expression. This delayed effect may be too long to reverse or inhibit post-cardiac arrest syndrome $(14,25)$. Second, a synergistic effect of heart injury and haemodynamic instability may reduce the tolerance of internal organs to ischemia $(26,27)$. Third, different times of drug administration may result in different effects. During 

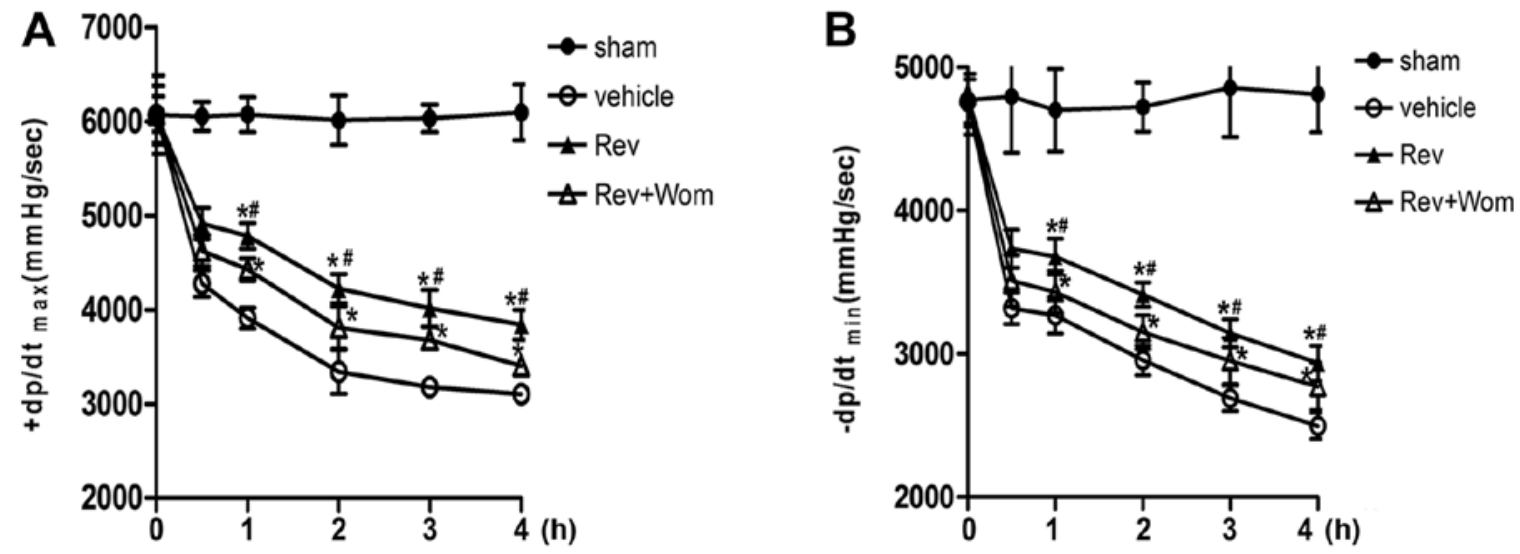

Figure 5. Left ventricular function. (A) $+\mathrm{dp} / \mathrm{dt}_{\max }$. (B) $-\mathrm{dp} / \mathrm{dt}_{\min }$. As previously mentioned, $+\mathrm{dp} / \mathrm{dt}_{\max }$ and $-\mathrm{dp} / \mathrm{dt}_{\min }$ in the vehicle group were lower compared to the sham group $(\mathrm{P}<0.05)$, while resveratrol protected left ventricular function $\left(\mathrm{P}<0.05 \mathrm{vs}\right.$. the vehicle group). $+\mathrm{dp} / \mathrm{dt}_{\max }$ and $-\mathrm{dp} / \mathrm{dt}_{\min }$ in the Rev + Wom group were lower compared to the Rev group $(\mathrm{P}<0.05)$, but higher compared to the vehicle group $(\mathrm{P}<0.05)$. ${ }^{*} \mathrm{P}<0.05$ vs. the vehicle group and ${ }^{\#} \mathrm{P}<0.05$ vs. the $2.3 \mathrm{mg} / \mathrm{kg}$ group. Rev, resveratrol; Wom, wortmannin.
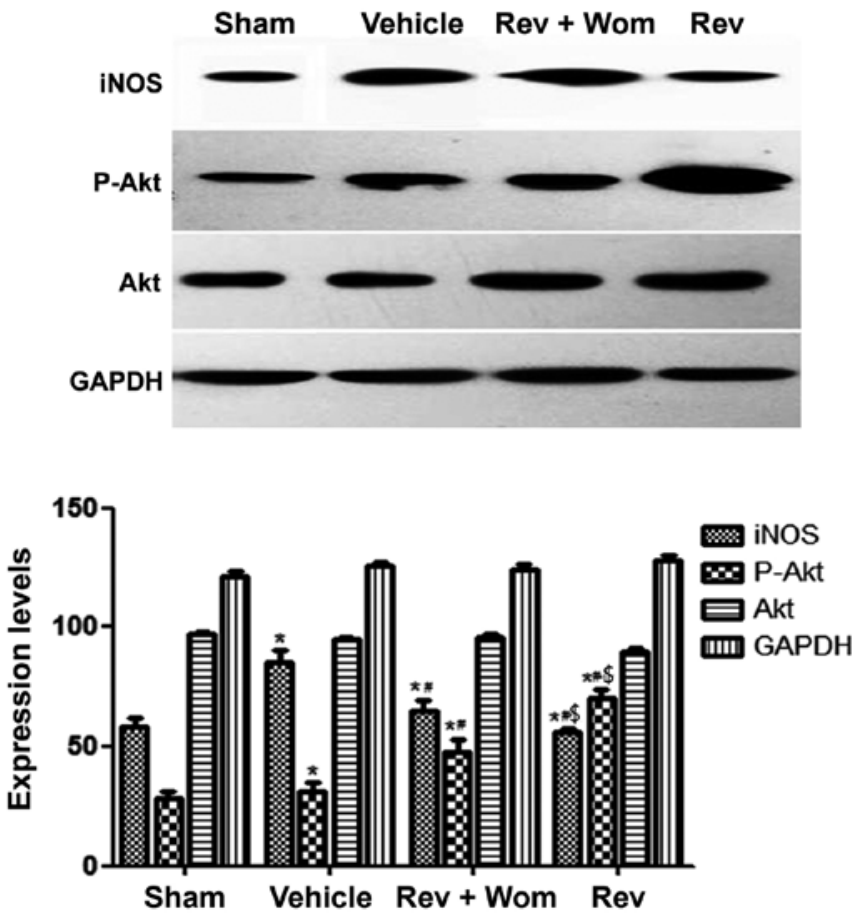

Figure 6. Representative blots of iNOS, p-Akt, and Akt expression in rat heart tissues from the different groups. Resveratrol decreased iNOS levels significantly while wortmannin partially reversed iNOS levels but were still lower compared to the vehicle group. Resveratrol increased p-Akt levels significantly while wortmannin completely blocked phosphorylation of Akt. No significant differences in total Akt levels were observed for the four groups. ${ }^{*} \mathrm{P}<0.05$ vs. the sham group, ${ }^{\prime} \mathrm{P}<0.05$ vs. the vehicle group and ${ }^{\mathrm{S}} \mathrm{P}<0.05$ vs. the Rev + Wom group. iNOS, inducible NOS; Rev, resveratrol; Wom, wortmannin.

CPR, chest compressions only provide blood volumes of $\sim 25-40 \%$ compared to normal stroke volume which may not attain effective drug concentration (28). In a rat CA model, administration of sevoflurane at the onset of ROSC rather than during resuscitation improved myocardial function (21). Finally, resveratrol was administered via bolus injection in the present study. Given the short half-life of resveratrol ( 8-14 min), whether continuous intravenous administration could improve myocardial function is yet to be demonstrated.
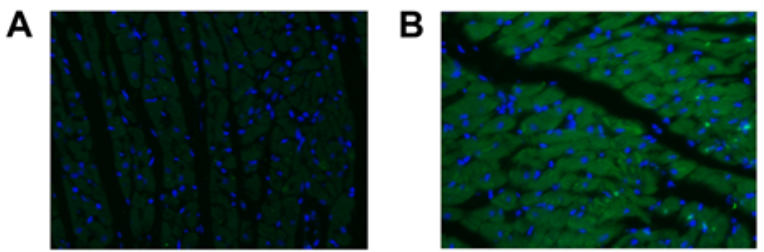

C
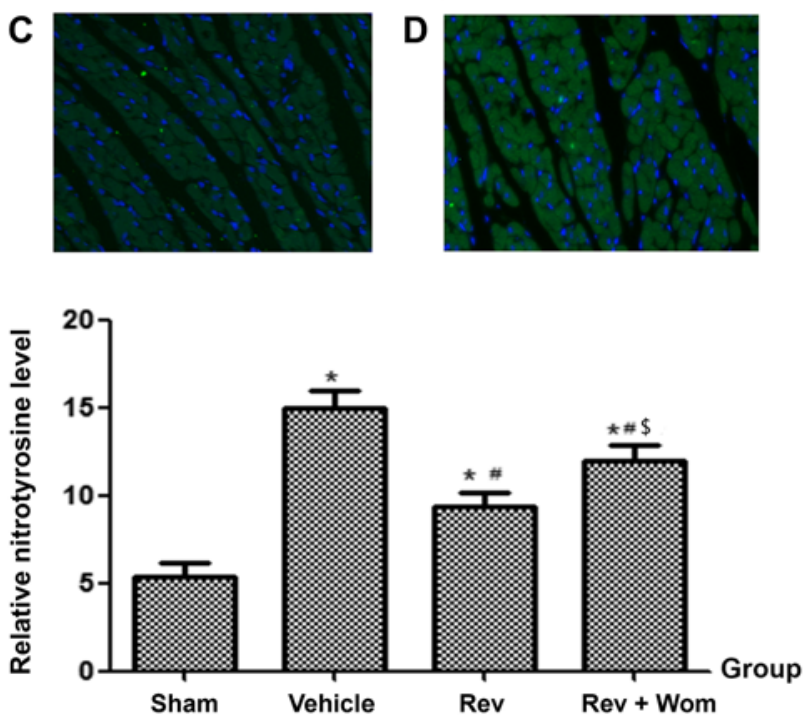

Figure 7. Representative photomicrographs of in situ detection of nitrotyrosine expression levels and DAPI staining in rat heart tissues (x400). (A) Sham; (B) vehicle; (C) resveratrol group; and (D) Rev + Wom group. Resveratrol significantly decreased nitrotyrosine expression levels, while wortmannin partially reversed this trend. ${ }^{*} \mathrm{P}<0.05$ vs. the sham group, ${ }^{\#} \mathrm{P}<0.05$ vs. the vehicle group and ${ }^{\$} \mathrm{P}<0.05$ vs. the Rev group. Rev, resveratrol; Wom, wortmannin.

Considering that the blood volume in rats is $\sim 100 \mathrm{ml} / \mathrm{kg}$ of its body weight, the calculated blood concentrations corresponding to the infused dose of resveratrol (MW:228.25) in the present study would be 100,10 and $1 \mu \mathrm{M}$. As previous studies revealed, resveratrol is characterized by hormesis, which means a stimulating effect at a lower level and an inhibitory effect at a higher level $(17,18)$. In our research, a lower level of resveratrol in fact revealed improved myocardial function than a higher level. The results were similar to a myocardial 
ischemia-reperfusion injury study involving resveratrol (29). However, we did not investigate whether lower doses would produce improved efficacy. A study by Hung et al (12), demonstrated that $2.3 \times 10^{-4} \mathrm{mg} / \mathrm{kg}$ resveratrol improved myocardial function in a rat myocardial infarction model. We hypothesize that resveratrol has selective distribution in vivo and hence plasma concentration does not represent concentration in the myocardium.

Myocardial nitrative stress after ROSC was similar to that reported previously in a mouse model (5). The authors observed that mitochondrial tyrosine nitration by peroxynitrite was significantly increased at $60 \mathrm{~min}$ after ROSC. In addition, it has been demonstrated that the extent of nitrative stress is proportional to the severity of myocardial dysfunction, with higher nitrative stress resulting from worse myocardial function (30). The results from our study indicated that myocardial nitrative stress was involved in the pathogenesis of myocardial dysfunction after ROSC.

The present results revealed that nitrative stress was involved in PCMD and resveratrol preconditioning ameliorated myocardial nitrative stress and decreased the expression of iNOS and nitrotyrosine. These results were similar to previous studies $(31,32)$ and suggested that resveratrol inhibits nitrative stress via a bidirectional regulation of the iNOS and eNOS expression levels. Resveratrol had a protective effect by downregulating excessive iNOS expression and upregulating endothelial NOS (eNOS) expression, with a concomitant minor increase in NO (31). However, there are several inconsistencies with previous studies. Resveratrol was revealed to induce iNOS expression in porcine pulmonary artery endothelial cells (33). The differences may be due to how the models were produced, experimental protocols, or which laboratory animals were used. It appears that lower expression of iNOS, rather than higher expression is important for resveratrol to exhibit its myocardial protective effect.

In the present study, wortmannin, a PI3K inhibitor, partially inhibited the myocardial protective effects of resveratrol by reducing p-Akt expression levels and increasing iNOS and nitrotyrosine levels. This suggests the PI3K/Akt signalling pathway was involved in the mechanism by which resveratrol inhibits nitrative stress. Our results were similar to previous studies. Wang et al (34) demonstrated that resveratrol exerts an anti-inflammatory effect by increasing the activity of adiponectin. It has been revealed that adiponectin enhanced eNOS phosphorylation and decreased ROS production, with a concomitant inhibition of nitrative stress via the PI3K/Akt signalling pathway in a mouse model of myocardial ischemia-reperfusion $(35,36)$. Thus, it was surmised that resveratrol can inhibit nitrative stress via adiponectin/PI3K/Akt, but this hypothesis requires validation in a further study. In a study by Huang et al (37), resveratrol inhibited oxygen-glucose deprivation-induced cell apoptosis via the NF- $\mathrm{B} / \mathrm{iNOS} / \mathrm{NO}$ signalling pathway. In an animal model of diabetic heart disease, resveratrol inhibited TNF- $\alpha$ and NF- $\kappa$ B expression, and thereby inhibited iNOS and ROS expression, which ameliorated nitrative stress and myocardial injury (38). From these studies, it is inferred that TNF- $\alpha$ and NF- $\mathrm{KB}$ are also involved in the mechanism by which resveratrol regulates iNOS expression via the PI3K/Akt signalling pathway in a cardiac arrest model. However, this needs to be established by further study.
A limitation of the present study is that healthy SD rats were used, which is not consistent with the clinical reality in which numerous CA victims have heart disease or are at a high risk for heart disease. SD rats are different from humans with regard to pathophysiological characteristics; hence, larger animals such as swine should be considered for future studies.

In addition, the study was conducted for only $4 \mathrm{~h}$ after ROSC, which is an appropriate early stage model for myocardial dysfunction after ROSC; however, going beyond $4 \mathrm{~h}$ after ROSC is necessary for investigating the effects of resveratrol on intermediate- and late-stage myocardial dysfunction.

The molecular mechanism of resveratrol as hypothesized in the present study is insufficient. The role of inflammatory factors in the inhibitory effect of resveratrol on nitrative stress should be investigated. In addition, as mentioned in several studies, mitochondria are critical for myocardial function after CA, and should be studied with regard to resveratrol.

Resveratrol preconditioning could alleviate cardiac dysfunction after resuscitation. This effect of resveratrol was associated with its inhibitory effect on nitrative stress and may partially involve the PI3K/Akt signalling pathway, however postconditioning with resveratrol was not more effective compared to the vehicle control.

\section{Acknowledgements}

The authors are grateful to Dr Si Rong He, Dr Hai Hu and Dr Ya Rong He from West China hospital of Sichuan University for their assistance.

\section{Funding}

The present study was partly supported by grants from the Support Project of Sichuan Science and Technology Department (2013SZ0061).

\section{Availability of data and materials}

The datasets used during the present study are available from the corresponding author upon reasonable request.

\section{Authors' contributions}

$\mathrm{ZZ}$ conceived and designed the study. $\mathrm{HZ}$ and QW performed the experiments. ZW and YC conducted the analysis of data. $\mathrm{HZ}$ wrote the manuscript and $\mathrm{ZZ}$ and $\mathrm{YC}$ revised the manuscript. All authors read and approved the final version of the manuscript.

\section{Ethics approval and consent to participate}

The present study was performed in strict accordance to the recommendations by the Guide for the Care and Use of Laboratory Animals, National Institutes of Health. The Institutional Animal Care and Use Committee of West China Hospital (Sichuan University; Approval no. 2016045A) approved the study protocol.

\section{Patient consent for publication}

Not applicable. 


\section{Competing interests}

The authors declare that they have no competing interests.

\section{References}

1. Hua W, Zhang LF, Wu YF, Liu XQ, Guo DS, Zhou HL, Gou ZP, Zhao LC, Niu HX, Chen KP, et al: Incidence of sudden cardiac death in China: Analysis of 4 regional populations. J Am Coll Cardiol 54: 1110-1118, 2009

2. Langhelle A, Tyvold SS, Lexow K, Hapnes SA, Sunde K and Steen PA: In-hospital factors associated with improved outcome after out-of-hospital cardiac arrest. A comparison between four regions in Norway. Resuscitation 56: 247-263, 2003.

3. Granfeldt A: Organ dysfunction following regional and global ischemia/reperfusion. Intervention with postconditioning and adenocaine. Dan Med J 59: B4496, 2012.

4. Adrie C, Adib-Conquy M, Laurent I, Monchi M, Vinsonneau C, Fitting C, Fraisse F, Dinh-Xuan AT, Carli P, Spaulding C, et al: Successful cardiopulmonary resuscitation after cardiac arrest as a 'sepsis-like' syndrome. Circulation 106: 562-568, 2002.

5. Han F, Da T, Riobo NA and Becker LB: Early mitochondria dysfunction in electron transfer activity and reactive oxygen species generation after cardiac arrest. Crit Care Med 36 (11 Suppl): S447-S453, 2008.

6. Brookes P and Darley-Usmar VM: Hypothesis: The mitochondrial NO $(*)$ signaling pathway, and the transduction of nitrosative to oxidative cell signals: An alternative function for cytochrome C oxidase. Free Radic Biol Med 32: 370-374, 2002.

7. Ischiropoulos $\mathrm{H}$ and Beckman JS: Oxidative stress and nitration in neurodegeneration: Cause, effect, or association? J Clin Invest 111: 163-169, 2003 .

8. Rossig L, Haendeler J, Hermann C, Malchow P, Urbich C, Zeiher AM and Dimmeler S: Nitric oxide down-regulates MKP-3 mRNA levels: Involvement in endothelial cell protection from apoptosis. J Biol Chem 275: 25502-25507, 2000.

9. Kim YM, Bombeck CA and Billiar TR: Nitric oxide as a bifunctional regulator of apoptosis. Circ Res 84: 253-256, 1999.

10. Jiao XY, Gao E, Yuan Y, Wang Y, Lau WB, Koch W, Ma XL and Tao L: INO-4885 [5,10,15,20-tetra[N-(benzyl-4'-carboxylate)2-pyridinium]-21H,23H-porphine iron(III) chloride], a peroxynitrite decomposition catalyst, protects the heart against reperfusion injury in mice. J Pharmacol Exp Ther 328: 777-784, 2009.

11. Arstall MA, Sawyer DB, Fukazawa R and Kelly RA: Cytokine-mediated apoptosis in cardiac myocytes: The role of inducible nitric oxide synthase induction and peroxynitrite generation. Circ Res 85: 829-840, 1999.

12. Hung LM, Chen JK, Huang SS, Lee RS and Su MJ: Cardioprotective effect of resveratrol, a natural antioxidant derived from grapes. Cardiovasc Res 47: 549-555, 2000.

13. Li D, Qu Y, Tao L, Liu H, Hu A, Gao F, Sharifi-Azad S, Grunwald Z, Ma XL and Sun JZ: Inhibition of iNOS protects the aging heart against beta-adrenergic receptor stimulation-induced cardiac dysfunction and myocardial ischemic injury. J Surg Res 131: 64-72, 2006.

14. Mokni M, Limam F, Elkahoui S, Amri M and Aouani E: Strong cardioprotective effect of resveratrol, a red wine polyphenol, on isolated rat hearts after ischemia/reperfusion injury. Arch Biochem Biophys 457: 1-6, 2007.

15. Shen M, Jia GL, Wang YM and Ma H: Cardioprotective effect of resvaratrol pretreatment on myocardial ischemia-reperfusion induced injury in rats. Vascul Pharmacol 45: 122-126, 2006.

16. Goh SS, Woodman OL, Pepe S, Cao AH, Qin C and Ritchie RH: The red wine antioxidant resveratrol prevents cardiomyocyte injury following ischemia-reperfusion via multiple sites and mechanisms. Antioxid Redox Signal 9: 101-113, 2007.

17. Hassan-Khabbar S, Cottart CH, Wendum D, Vibert F, Clot JP, Savouret JF, Conti M and Nivet-Antoine V: Postischemic treatment by trans-resveratrol in rat liver ischemia-reperfusion: A possible strategy in liver surgery. Liver Transpl 14: 451-459, 2008.

18. Gurusamy N, Lekli I, Mukherjee S, Ray D, Ahsan MK Gherghiceanu M, Popescu LM and Das DK: Cardioprotection by resveratrol: A novel mechanism via autophagy involving the mTORC2 pathway. Cardiovasc Res 86: 103-112, 2010

19. Chen MH, Liu TW, Xie L, Song FQ, He T, Mo SR and Zeng ZY: A simpler cardiac arrest model in the mouse. Resuscitation 75 372-379, 2007
20. Yin XL, Zhang W, Yang Y and Shen H: Increasing expression of (CCAAT enhancer binding protein) homologous protein induced by endoplasmic reticulum stress in myocardium after cardiac arrest and resuscitation in rat. Resuscitation 83: 378-385, 2012.

21. Knapp J, Bergmann G, Bruckner T, Russ N, Bottiger BW and Popp E: Pre- and postconditioning effect of sevoflurane on myocardial dysfunction after cardiopulmonary resuscitation in rats. Resuscitation 84: 1450-1455, 2013.

22. Mukherjee S, Dudley JI and Das DK: Dose-dependency of resveratrol in providing health benefits. Dose Response 8: 478-500, 2010.

23. Shen M, Wu RX, Zhao L, Li J, Guo HT, Fan R, Cui Y, Wang YM, Yue SQ and Pei JM: Resveratrol attenuates ischemia/reperfusion injury in neonatal cardiomyocytes and its underlying mechanism. PLoS One 7: e51223, 2012

24. Boocock DJ, Faust GE, Patel KR, Schinas AM, Brown VA Ducharme MP, Booth TD, Crowell JA, Perloff M, Gescher AJ, et al: Phase I dose escalation pharmacokinetic study in healthy volunteers of resveratrol, a potential cancer chemopreventive agent. Cancer Epidemiol Biomarkers Prev 16: 1246-1252, 2007.

25. Juan SH, Cheng TH, Lin HC, Chu YL and Lee WS: Mechanism of concentration-dependent induction of heme oxygenase-1 by resveratrol in human aortic smooth muscle cells. Biochem Pharmacol 69: 41-48, 2005

26. Safar P: Effects of the postresuscitation syndrome on cerebral recovery from cardiac arrest. Crit Care Med 13: 932-935, 1985.

27. Senthil M, Brown M, Xu DZ, Lu Q, Feketeova E and Deitch EA Gut-lymph hypothesis of systemic inflammatory response syndrome/multiple-organ dysfunction syndrome: Validating studies in a porcine model. J Trauma 60: 958-967, 2006.

28. Rubertsson S, Grenvik A and Wiklund L: Blood flow and perfusion pressure during open-chest versus closed-chest cardiopulmonary resuscitation in pigs. Crit Care Med 23: 715-725, 1995.

29. Bradamante S, Barenghi L, Piccinini F, Bertelli AA, De Jonge R, Beemster P and De Jong JW: Resveratrol provides late-phase cardioprotection by means of a nitric oxide- and adenosinemediated mechanism. Eur J Pharmacol 465: 115-123, 2003.

30. Wu D, Bassuk J, Arias J, Kurlansky P, Lozano H, Lamas G and Adams JA: Different roles of nitric oxide synthase isoforms in cardiopulmonary resuscitation in pigs. Resuscitation 73: 144-153, 2007.

31. Hung LM, Su MJ and Chen JK: Resveratrol protects myocardial ischemia-reperfusion injury through both NO-dependent and NO-independent mechanisms. Free Radic Biol Med 36: 774-781, 2004.

32. Zhang HX, Duan GL, Wang CN, Zhang YQ, Zhu XY and Liu YJ: Protective effect of resveratrol against endotoxemia-induced lung injury involves the reduction of oxidative/nitrative stress. Pulm Pharmacol Ther 27: 150-155, 2014.

33. Hsieh TC, Juan G, Darzynkiewicz Z and Wu JM: Resveratrol increases nitric oxide synthase, induces accumulation of p53 and p21 (WAF1/CIP1), and suppresses cultured bovine pulmonary artery endothelial cell proliferation by perturbing progression through S and G2. Cancer Res 59: 2596-2601, 1999.

34. Wang A, Liu M, Liu X, Dong LQ, Glickman RD, Slaga TJ, Zhou Z and Liu F: Up-regulation of adiponectin by resveratrol: The essential roles of the Akt/FOXO1 and AMP-activated protein kinase signaling pathways and DsbA-L. J Biol Chem 286: 60-66, 2011.

35. Ji L, Fu F, Zhang L, Liu W, Cai X, Zhang L, Zheng Q, Zhang H and Gao F: Insulin attenuates myocardial ischemia/reperfusion injury via reducing oxidative/nitrative stress. Am J Physiol Endocrinol Metab 298: E871-E880, 2010.

36. Wang WQ, Zhang HF, Gao GX, Bai QX, Li R and Wang XM: Adiponectin inhibits hyperlipidemia-induced platelet aggregation via attenuating oxidative/nitrative stress. Physiol Res 60: 347-354, 2011.

37. Huang T, Gao D, Jiang X, Hu S, Zhang L and Fei Z: Resveratrol inhibits oxygen-glucose deprivation-induced MMP-3 expression and cell apoptosis in primary cortical cells via the NF-kappaB pathway. Mol Med Rep 10: 1065-1071, 2014.

38. Zhang H, Morgan B, Potter BJ, Ma L, Dellsperger KC, Ungvari Z and Zhang C: Resveratrol improves left ventricular diastolic relaxation in type 2 diabetes by inhibiting oxidative/nitrative stress: In vivo demonstration with magnetic resonance imaging. Am J Physiol Heart Circ Physiol 299: H985-H994, 2010.

This work is licensed under a Creative Commons Attribution-NonCommercial-NoDerivatives 4.0 International (CC BY-NC-ND 4.0) License. 